\title{
Efeito do laser infravermelho de baixa potência na osseointegração pós-implante: revisão de literatura
}

\author{
Effect of low-power infrared laser in post-implant osseintegration: literature review \\ Efecto del laser infrarrojo de baja potencia en la osseointegración post-implante: revisão \\ de literatura
}

Sabrina Thais Ferreira Cardoso Munhoz ${ }^{1 *}$, Gabrielly Lopes Correia Vergeth de Sirqueira ${ }^{1}$, Lucas Fortes Cavalcanti de Macêdo'.

\section{RESUMO}

Objetivo: O objetivo deste trabalho foi, através de uma revisão de literatura, discutir e analisar as características do laser de baixa potência e seus efeitos terapêuticos sobre os tecidos nas áreas pósimplantadas, bem como abranger protocolos e usabilidade clínica. Métodos: Foi realizado uma busca guiada nas línguas portuguesa e inglesa no período de 2000 a 2019 através dos termos disponibilizados no DeCS e textos nas bases de dados Scielo, PubMed, Scholar Google, Medline e Lilacs. Resultados: A implantodontia é uma especialidade dentro da odontologia responsável por reestabelecer função mastigatória e estética de espaços edêntulos, visando substituir por implante sítios onde houveram perdas dentais. Para que um implante seja bem-sucedido, é necessário que o fenômeno da osseointegração aconteça, o qual é influenciado por condições locais e sistêmicas que podem interferir na sobrevida do implante em função. Visando esse aspecto, a utilização do laser de baixa potência é um avanço que pode favorecer a reabilitação pós-cirúrgica e acelerar o processo de osseointegração. Considerações Finais: Embora os lasers de baixa intensidade apresentem bons resultados, sua eficácia é pouco comprovada no que se refere ao reparo do tecido ósseo, se mostrando um excelente coadjuvante no tratamento de tecidos moles e procedimentos terapêuticos, apenas.

Palavras-chave: Reabilitação Bucal, Terapia com Luz de Baixa Intensidade, Osseointegração.

\begin{abstract}
Objective: The objective of this work was to discuss and analyze the characteristics of low-power laser and its therapeutic effects on tissues in post-implanted areas, as well as protocols and clinical usability. Methods: A guided search in the Portuguese and English languages was conducted from 2000 to 2019 using the terms provided in the DeCS and texts in the databases Scielo, PubMed, Scholar Google, Medline and Lilacs. Results: Implant dentistry is a specialty within the dentistry responsible for reestablishing masticatory and aesthetic functions of edentulous spaces, aiming to replace dental implants with dental implants. For an implant to be successful, it is necessary for the osseointegration phenomenon to occur, which is influenced by local and systemic conditions that may interfere with implant-to-implant survival. Aiming at this aspect, the use of low-power laser is an advance that may favor post-surgical rehabilitation and accelerate the osseointegration process. Final Thoughts: Although low intensity lasers have good results, their efficacy is poorly demonstrated for bone tissue repair, being an excellent adjunct in soft tissue treatment and therapeutic procedures only.
\end{abstract}

Key words: Mouth Rehabilitation, Low Intensity Light Therapy, Osseointegration.

${ }^{1}$ Centro Universitário Cesmac, Maceió-AL. *E-mail: licyathaynas@hotmail.com

SUBMETIDO EM: 6/2019 


\section{RESUMEN}

Objetivo: El objetivo de este trabajo fue discutir y analizar las características del láser de baja potencia y sus efectos terapéuticos en los tejidos en áreas postimplantadas, así como los protocolos y la facilidad de uso clínico. Métodos: se realizó una búsqueda guiada en los idiomas portugués e inglés de 2000 a 2019 utilizando los términos proporcionados en el DeCS y los textos en las bases de datos Scielo, PubMed, Scholar Google, Medline y Lilacs. Resultados: la odontología de implantes es una especialidad dentro de la odontología responsable del restablecimiento de las funciones estéticas y masticatorias de los espacios edéntulos, con el objetivo de reemplazar los implantes dentales con implantes dentales. Para que un implante tenga éxito, es necesario que ocurra el fenómeno de oseointegración, que está influenciado por condiciones locales y sistémicas que pueden interferir con la supervivencia del implante al implante. Apuntando a este aspecto, el uso de láser de baja potencia es un avance que puede favorecer la rehabilitación postquirúrgica y acelerar el proceso de osteointegración. Pensamientos finales: Aunque los láseres de baja intensidad tienen buenos resultados, su eficacia está pobremente demostrada para la reparación del tejido óseo, ya que es un complemento excelente en el tratamiento de tejidos blandos y en los procedimientos terapéuticos únicamente.

Palabras Clave: Rehabilitacion Oral, Terapia de luz de baja intensidad, Osteointegración.

\section{INTRODUÇÃO}

A implantodontia é uma especialidade dentro da odontologia responsável por reestabelecer função mastigatória e estética de espaços edêntulos, visando substituir por implante sítios onde houveram perdas dentais (HADDAD MF, et al., 2008) que, comumente como especialidade evolui de forma rápida na busca de técnicas para que o implante se torne uma alternativa de maior garantia, previsibilidade e conforto na odontologia (FRIGGI TR, et al., 2011).

Para que um implante seja bem-sucedido, é necessário que o fenômeno da osseointegração aconteça, isto é, a união física do implante osseointegrado com o osso receptor idealizada por Branemark em 1954 (MARTINS V et al., 2011). No entanto, o processo é influenciado por condições locais e sistêmicas que podem interferir na sobrevida do implante em função (MIRANDA V, et al., 2018).

Dentre as técnicas terapêuticas para minimizar ou evitar danos aos implantes bem como favorecer a osseointegração, é possível citar o laser de baixa potência tendo em vista seu potencial de acelerar a reparação do tecido ósseo peri-implantar e biomodular os processos inflamatórios (FRIGGI TR, et al., 2011).

A utilização do laser de baixa potência é considerada um dos maiores avanços tecnológicos para a medicina e a odontologia, o qual comumente é empregado em alguns tipos de diagnóstico e terapias coadjuvantes (GENOVESE WJ, 2007; JESUS LK, 2017).

O laser de baixa intensidade (LBI) emite radiações sem potencial destrutivo e com efeitos terapêuticos sobre os tecidos. Quando utilizado associado a corantes tem o potencial de produzir morte de microrganismos em um processo conhecido como terapia fotodinâmica (YAMADA JÚNIOR A, HAYEK R, RIBEIRO M, 2004; JESUS LK, 2017).

As terapias com laser de baixa intensidade, denominadas de fotobiomodulação, são responsáveis por diferentes efeitos na modulação celular, incluindo aceleração do reparo de feridas, aumento da taxa de metabolismo celular e tratamento não-invasivo de numerosas patologias relacionadas à disfunção mitocondrial (GENOVESE WJ, 2007).

A aplicação dos diferentes tipos de lasers na odontologia possibilitou avanços nos procedimentos por proporcionar grande diminuição do tempo trans-cirúrgico e de recuperação pós-cirúrgica, diminuição de complicações pós-operatórias, redução de sinais clínicos flogísticos e, ainda, bioestimulação de tecidos moles e ósseos, bem como maior controle analgésico de dores crônicas (XAVIER KCR, SCHMIDT EMM, SILVA G, 2008; JESUS LK, 2017). 
O objetivo deste trabalho é, através de uma revisão de literatura, discutir e analisar as características do laser de baixa potência e seus efeitos terapêuticos sobre os tecidos nas áreas pós-implantadas, bem como abranger protocolos e usabilidade clínica satisfatórios.

\section{METODOLOGIA}

Este trabalho é uma revisão de literatura sistemática quantitativa descritiva, e para a elaboração foram consultados trabalhos de revisão e/ou pesquisa publicados em periódicos científicos em línguas portuguesa e inglesa no período de 2000 a 2019 disponibilizados nas bases de dados Scielo, PubMed, Scholar Google, Medline e Lilacs.

A busca foi realizada a partir dos descritores "Reabilitação Bucal", "Terapia com Luz de Baixa Intensidade" e "Osseointegração", indexados nos Descritores e Ciências da Saúde. Através do cruzamento das palavraschave, foi possível colher uma amostra de 41 artigos científicos relevantes ao tema, sendo excluídos resumos ou trabalhos não disponíveis na íntegra ou não disponíveis nas línguas de escolha inicial.

Destes trabalhos, 21 foram selecionados para compor a revisão levando em consideração as características do laser de baixa potência, sua usabilidade e efeitos terapêuticos, bem como exemplos de protocolos clínicos satisfatórios em concordância com a literatura.

\section{RESULTADOS}

\section{O uso do laser}

A palavra LASER é um acrônimo de "Light Amplification by Stimulated Emission of Radiation" que se traduz em "amplificação da luz por emissão estimulada de radiação" (CASTILHO FT, 2003; PROCKT P, TAKAHASHI A, PAGNONCELLI RM, 2008; LUÍS AA, 2013).

Trata-se de um dispositivo que produz um feixe de luz especial comumente chamado de "raio laser", dividido em laseres de baixa potência e laseres cirúrgicos (LINS RDAU et al., 2011).

Foi em 1916 que Albert Einstein traçou os princípios de geração e desenvolvimento dos lasers quando formulou a Teoria da Emissão Estimulada de Radiação (PROCKT P, TAKAHASHI A, PAGNONCELLI RM, 2008; LUÍS AA, 2013). Mas, o emprego do raio laser no processo de reparação tecidual foi estudado pela primeira vez em 1971 por Mester et al., quando observaram que feridas em ratos cicatrizavam mais rápido sob a ação do laser terapêutico (LINS RDAU et al., 2011).

O laser difere da luz comum em relação ao seu direcionamento: a comum é propagada de maneira difusa e o laser é propagado de maneira retilínea. Isso acontece porque o laser apresenta um único comprimento de onda, diferente da luz comum e, por isso, é chamado de monocromático (CASTILHO FT, 2003), característica a qual se refere à especificidade da luz de um único comprimento de onda definido (LUíS AA, 2013).

Por si só, essa característica permite a interação com tecidos biológicos e promove desde efeitos terapêuticos a cirúrgicos de corte, coagulação por cauterização ou métodos diagnósticos, a depender da intensidade (CASTILHO FT, 2003).

O comprimento da onda é o fator crítico na determinação dos efeitos produzidos pelo laser, já que esse parâmetro determina quais biomoléculas específicas serão absorvidas na radiação incidente e qual a interação fotobiológica básica por trás de um determinado efeito de tratamento (LUÍS AA, 2013).

Geralmente, a profundidade tecidual alcançada pela laserterapia de baixa potência é sempre proporcional a parâmetros físicos como a potência, o comprimento de onda e o tempo de exposição. Quanto maior a onda, maior a capacidade de penetração. A quantidade de energia aplicada é expressa em Joules sobre a área expressa $\mathrm{em} \mathrm{cm}^{2} \mathrm{e}$, comumente, a absorção da luz depende da densidade tecidual, dureza, maleabilidade e pigmentação (FLORENTIN ACA et al., 2015). 
O desenvolvimento de diversos tipos de laser e vários comprimentos de onda possibilitou seu uso em diversos procedimentos terapêuticos (PROCKT P, TAKASHASHI A, PAGNONCELLI RM, 2008). Quando aplicada em doses apropriadas, a energia luminosa do laser pode estimular aumento na produção de linfócitos, ATP mitocondrial e fibroblastos, promovendo efeitos terapêuticos (LINS RDAU et al., 2011).

Dentre os principais efeitos terapêuticos estão: ação anti-inflamatória e analgésica, prevenção de infecções e aceleração nas fases iniciais do processo de cicatrização em tecidos biológicos, o que sugere possível irradiação ao tecido ósseo submetido a implantes e promoção da aceleração no processo de osseointegração, levando a uma diminuição do tempo requerido para osseointegração, o que ainda não está bem esclarecido (FREITAS IGF, BARANAUSKAS V, CRUZ HM, 2000; GUZZARDELLA GA et. al. 2001; LINS RDAU et al., 2011; ZANCA MM et al., 2016).

\section{Osseointegração x LBI}

O uso de implantes osseointegrados representou um grande avanço na perspectiva da reabilitação do edentulismo parcial e/ou total (TORRES MARO e TEIXEIRA ER, 2008) e seu sucesso na instalação se relacionou diretamente com a correta carga oclusal, material utilizado, desenho, superfície do implante, estabilidade primária, osseointegração e técnica cirúrgica (BRUNETTO JL et al., 2016; SILVA FL et al., 2016).

Dentre esses fatores, o processo de osseointegração, isto é, a formação de tecido ósseo ao redor do implante, é determinante para o sucesso da reabilitação protética de rebordos edêntulos com implantes dentários (BRUNETTO JL et al., 2016; SILVA FL et al., 2016), onde topografia, molhabilidade, carga de superfície e composição química superficial, em contato com o tecido ósseo, definem velocidade e qualidade dessa característica (SILVA FL et al., 2016).

O LBI, em implantodontia, frequentemente é indicado para a descontaminação de superfície de implantes, tratamento de periimplantites, diminuição de edema, dor e inflamação pós-operatória, em procedimentos de segundo estágio cirúrgico de implantes submersos (incisão/excisão), cirurgia plástica de tecidos moles e, de maneira experimental, durante o preparo do leito ósseo previamente à instalação do implante (FARIAS IOB e FREITAS MA, 2017).

Considerando seus efeitos terapêuticos, sua aplicabilidade na aceleração da reparação óssea demonstrou resultados positivos e proporcionou um maior contato osso-implante (CESTARI MN, 2017).

Dinato JC e Polido WD (2001) observaram que o uso do laser como estimulador da osseointegração é favorável. A aceleração no reparo do tecido ósseo pode ser vista tanto clínica como histologicamente quando comparada a técnicas convencionais pós-cirúrgicas. É nítido que o uso do laser de baixa potência proporciona um melhor reparo do tecido ósseo ao redor do implante, redução de edema e diminuição da dor.

Já DÖRTBUDAK O et al. (2002) observaram o efeito do laser "in vivo" em 15 pacientes implantados e constataram que a utilização do laser de baixa potência reduz grupos de bactérias, mas, não osseointegração com o comprimento de onda de $690 \mathrm{mn}$ por 60 segundos, irradiada com laser de diodo suave.

JAKSE $\mathrm{N}$ et al. (2007) mostraram que a influência do laser de baixa intensidade sobre a regeneração óssea e osseointegração de implantes após a cirurgia de levantamento de seio maxilar, apresenta melhor osseointegração dos implantes, mas não diferença significativa na formação óssea do enxerto.

Um pouco mais a frente, visando avaliar os efeitos do LBI no processo de osseointegração de implantes com superfície usinada, CESTANARI MN (2017) instalou implantes na tíbia de coelhos e, como resultado, comprovou que a utilização do LBI acelerou as fases iniciais do processo e permitiu valores de torque de remoção e estabilidade superiores aos não expostos pela laserterapia.

Apesar de o tema estar sendo mais explorado com o passar dos anos, a utilização do LBI não é recente e, os efeitos terapêuticos (bioestimulação) comprovadamente promovem melhor controle de reações inflamatórias e sangramento, aceleração dos processos de cicatrização, regeneração óssea, restabelecimento da função neural, atenuação dos processos dolorosos, potencialização do reparo de defeitos ósseos e fraturas, aceleração da maturação óssea, aumento das atividades dos macrófagos e 
deposição de cálcio a depender do comprimento de onda e exposição (BLAY A, 2001; MARIA MMA, 2015; FARIAS IOB e FREITAS MA, 2017). No entanto, resultados negativos relacionados à regeneração óssea existem. BERGAMASCHI IP (2016) perfurou e preencheu a tíbia de 20 coelhos com osso liofilizado bovino e membrana de colágeno e os submeteu à terapia com LBI. Não constatou efeito da terapia laser sistêmica sobre o processo de reparação óssea. Apesar de existirem estudos com resultados positivos sobre a aceleração da regeneração óssea que o LBI reproduz (DINATO JC e POLIDO WD, 2001; DÖRTBUDAK O et al., 2002; JAKSE $N$ et al., 2007; CESTANARI MN, 2017), se comparados ao processo de reparação dos tecidos moles, os dados obtidos acerca da utilização dos laseres para reparo ósseo ainda é bastante incipiente (GENOVESE WJ, 2000).

\section{Protocolos}

Os LBI têm sido muito utilizados na Odontologia no que se refere ao processo reparativo, provavelmente pela sua ação analgésica e anti-inflamatória, o que o torna uma alternativa complementar de tratamento (GARCIA VG et al., 2001; LINS RDAU et al., 2011; ZANCA MM et al., 2016).

Dada a necessidade da osseointegração em implantodontia, GENOVESE WJ et al. (2007) formularam protocolos e finalidades visando diminuir o tempo de espera (Quadro 1).

Quadro 1 - Protocolos adaptados de Genovese WJ et al., (2007), São Paulo.

\begin{tabular}{|c|c|c|}
\hline \multicolumn{2}{|c|}{ Protocolos para a utilização de laseres na implantodontia } & Finalidade \\
\hline $\begin{array}{l}\text { Acelerador do } \\
\text { processo de } \\
\text { reparação óssea }\end{array}$ & $\begin{array}{l}\text { Antes da colocação dos implantes, os alvéolos cirúrgicos } \\
\text { deverão ser irradiados perpendicularmente com } 3 \text { a } \\
4 \mathrm{~J} / \mathrm{cm}^{2} \text {. Após a realização da sutura, aplica-se de } 6 \text { a } \\
8 \mathrm{~J} / \mathrm{cm}^{2} \text { em varredura. }\end{array}$ & $\begin{array}{l}\text { Bioestimulação, } \\
\text { analgesia, efeito anti- } \\
\text { inflamatório e anti- } \\
\text { edematoso. }\end{array}$ \\
\hline Exertos ósseos & $\begin{array}{l}\text { Imediatamente após a instalação do enxerto, a área deve } \\
\text { ser irradiada com movimentos circulares de varredura } \\
\text { com dosagem de } 6 \text { a } 8 \mathrm{~J} / \mathrm{cm}^{2} \text {, em todo o enxerto, após a } \\
\text { sutura aplicar a mesma dosagem só que em movimentos } \\
\text { de varredura. }\end{array}$ & $\begin{array}{l}\text { Aumento de velocidade } \\
\text { de formação óssea, } \\
\text { analgesia, indução óssea } \\
\text { e efeito anti-inflamatório. }\end{array}$ \\
\hline $\begin{array}{l}\text { Cirurgia de } \\
\text { levantamento de } \\
\text { seio maxilar }\end{array}$ & $\begin{array}{l}\text { A aplicação do laser se inicia antes da anestesia local, } \\
\text { aplicando-se em movimento de varredura no local da } \\
\text { cirurgia de } 6 \text { a } 8 \mathrm{~J} / \mathrm{cm}^{2} \text {. Antes do enxerto ósseo, aplica- } \\
\text { se uma densidade energética de } 3 \text { a } 4 \mathrm{~J} / \mathrm{cm}^{2} \text {, após a } \\
\text { colocação do enxerto, volta-se a aplicar a mesma } \\
\text { densidade energética sobre este enxerto. Após a sutura } \\
\text { deve-se aplicar de } 6 \text { a } 8 \mathrm{~J} / \mathrm{cm}^{2} \text { sobre a mesma, com } \\
\text { movimento de varredura. }\end{array}$ & $\begin{array}{l}\text { Bioestimulação, } \\
\text { analgesia, efeito anti- } \\
\text { inflamatório e anti- } \\
\text { edematoso. }\end{array}$ \\
\hline $\begin{array}{l}\text { Cirurgia de } \\
\text { aumento de } \\
\text { rebordo (enxerto } \\
\text { em bloco) }\end{array}$ & $\begin{array}{l}\text { Repete-se o protocolo anterior com uma diferença: no } \\
\text { interior da área doadora, será aplicado } 4 \mathrm{~J} / \mathrm{cm}^{2} \text {, e após a } \\
\text { sutura, } 8 \mathrm{~J} / \mathrm{cm}^{2} \mathrm{em} \text { movimento de varredura. }\end{array}$ & $\begin{array}{l}\text { Bioestimulação, } \\
\text { analgesia, efeito anti- } \\
\text { inflamatório e anti- } \\
\text { edematoso. }\end{array}$ \\
\hline $\begin{array}{l}\text { Laterização do } \\
\text { nervo alveolar }\end{array}$ & $\begin{array}{l}\text { Aplica-se na região a ser operada } 8 \mathrm{~J} / \mathrm{cm}^{2} \text { antes da } \\
\text { anestesia e } 4 \mathrm{~J} / \mathrm{cm}^{2} \text { nos seguintes passos de aplicação da } \\
\text { energia a laser: sobre o nervo alveolar exposto, no } \\
\text { interior dos alvéolos artificiais, sobre o nervo repousado } \\
\text { nos implantes e após o tamponamento da fenestração. } \\
\text { Posteriormente, de } 6 \text { a } 8 \mathrm{~J} / \mathrm{cm}^{2} \text { sobre a sutura. }\end{array}$ & $\begin{array}{l}\text { Bioestimulação, } \\
\text { analgesia, efeito anti- } \\
\text { inflamatório e anti- } \\
\text { edematoso e reparação } \\
\text { do nervo manipulado, } \\
\text { evitando parestesia. }\end{array}$ \\
\hline $\begin{array}{l}\text { Distração } \\
\text { Osteogênica }\end{array}$ & $\begin{array}{l}\text { Em todas as fases da cirurgia, em forma de varredura, de } \\
6 \text { a } 8 \mathrm{~J} / \mathrm{cm}^{2} \text { sobre a área que vai ser operada, ao final da } \\
\text { primeira cirurgia e na sutura. }\end{array}$ & $\begin{array}{l}\text { Bioestimulação, } \\
\text { analgesia, efeito anti- } \\
\text { inflamatório e anti- } \\
\text { edematoso. }\end{array}$ \\
\hline Parestesia & $\begin{array}{l}4 \mathrm{~J} / \mathrm{cm}^{2} \text { nos pontos de saída dos nervos e na trajetória } \\
\text { das fibras nervosas. }\end{array}$ & $\begin{array}{l}\text { Bioestimulação, } \\
\text { analgesia, efeito anti- } \\
\text { inflamatório e anti- } \\
\text { edematoso. }\end{array}$ \\
\hline
\end{tabular}

*: Tempo de repetição do protocolo: 24 horas, 48 horas e 72 horas. Fonte: Genovese et. al. (2007). 
Por sua vez, a terapia promove mudanças de caráter metabólico, energético e funcional nos tecidos submetidos à radiação, o que resulta na evolução para a cura em um período de tempo menor (BERGAMASCHI IP, 2016).

Estes laseres não apresentam um potencial destrutivo e sua atuação terapêutica e biomoduladora se dá através de uma ação fotoquímica que, por sua vez, estimula: aumento de fibroblastos, regeneração de vasos sanguíneos, velocidade de reparação dos vasos seccionados, reepitelização, ritmo de divisão celular (GENOVESE WJ, 2000), atividade osteoblástica e um trabeculado ósseo mais proeminente, bem como maior resistência à fratura e dureza estrutural (PROCKT P, TAKASHASHI A, PAGNONCELLI RM, 2008).

Trata-se de uma energia monocromática, coerente e direcionada que estimula a ativação dos componentes da cadeia respiratória em cascata e que promove proliferação celular e citoproteção, resultado da ação de radicais livres que induzem a ativação celular (PROCKT P, TAKAHASHI A, PAGNONCELLI RM, 2008).

No entanto, os efeitos dos LBI no tecido ósseo ainda são controversos (PIRES-OLIVEIRA DDA et al., 2010). Não existem protocolos universalmente aceitos com o objetivo da promoção de regeneração óssea ou osseointegração. Todos divergem em parâmetros como tipo de laser, comprimento de onda, densidade energética e tempo de aplicação (PIRES ODDA et al., 2010; MARIA MMA, 2015).

\section{CONSIDERAÇÕES FINAIS}

O presente estudo permite concluir que, embora os LBI apresentem bons resultados no que remete sua ação terapêutica em tecidos moles e controle analgésico e inflamatório, sua eficácia em relação à osseointegração e reparação óssea são duvidosas e requerem mais pesquisas na área, tendo como perspectiva que os tecidos irradiados obtenham auxilio em seu processo inflamatório e em sua reparação cicatricial. Ainda que os estudos sugeriram diferentes tipos de laseres e terapias coadjuvantes, bem como protocolos diferenciados para este fim, ainda não existe um protocolo universal e trabalhos sobre seu uso visando a regeneração não se mostram tão favoráveis.

\section{REFERÊNCIAS}

1. BERGAMASCHI IP. Avaliação do efeito sistêmico do laser de baixa intensidade sobre o processo de reparo ósseo com biomateriais: estudo em coelhos. 2016. 50 f. Trabalho de Conclusão de Curso (Monografia). Universidade Federal do Rio Grande do Sul, Faculdade de Odontologia, Porto Alegre, 2016.

2. BLAY A. Efeitos da radiação laser em baixa intensidade no mecanismo de osseointegração de implantes: estudo in vivo. 2001. 135 f. Trabalho de Conclusão de Curso (Dissertação de Mestrado). Instituto de Pesquisas Energéticas Nucleares, Autarquia Associada à Universidade de São Paulo, São Paulo, 2001.

3. BRUNETTO JL. et al. A influência da microgeometria do implante na osseointegração - revisão de literatura. Revista Uningá Review, Uningá, v. 26, n. 1, p. 52-32, 2016.

4. CASTILHO FT. Avaliação biomecânica da ação da radiação laser em baixa intensidade no processo de osseointegração de implantes de titânio inseridos em tíbia de coelhos. 2003. 71 f. Trabalho de Conclusão de Curso (Dissertação de Mestrado). Instituto de Pesquisas Energéticas Nucleares, Autarquia Associada à Universidade de São Paulo, São Paulo, 2003.

5. CESTARI MN. Efeito da laserterapia de baixa intensidade na osseointegração de implantes de superfície lisa. 2017. 43 f. Trabalho de Conclusão de Curso (Monografia). Universidade Estadual Paulista "Júlio de Mesquita Filho", Faculdade de Odontologia de Araçatuba, Araçatuba, 2017.

6. DINATO JC.; POLIDO, WD. Implantes osseointegrados: cirurgia e prótese. 1.ed. São Paulo: Artes Médicas, 2001.

7. DÖRTBUDAK O. et al. Lethal photosensitization for decontamination of implant surfaces in the treatment of periimplantitis. Clinical Oral Implants Research, Paris, v. 12, n. 1, p. 104-108, 2001.

8. FARIAS IOB.; FREITAS, MA. Aplicação do laser no tratamento da periimplantite. Revista Bahiana de Odontologia, Salvador, v. 8, n. 4, p. 141-151, 2017.

9. FLORENTIN ACA. Tratamento da mucosite oral com laser de baixa potência: revisão sistemática de literatura. Revista de Ciências Médicas, Campinas, v. 24, n. 2, p. 85-92, 2015.

10. FREITAS, IGF.; BARANAUSKAS V.; CRUZ-HÖFLING, M. A. Laser effects on osteogenesis. Applied Surface Science, Amsterdã, v. 154, n. 1, p. 548-554, 2000. 
11. FRIGGI TR. et al. Laserterapia aplicada à implantodontia: análise comparativa entre diferentes protocolos de irradiação. Innovations Implant Journal: Biomaterials and Esthetics (Online), São Paulo, v. 6, n. 1, p. 44-48, 2011.

12. GARCIA VG. et al. Processo de reparo em feridas de extração dental tratadas com laser em baixa intensidade $(904 \mathrm{~nm})$, com diferentes energias de irradiação. Estudo histológico em ratos. Revista da Faculdade de Odontologia de Lins/UNIMEP, Lins, v. 13, n. 2, p. 27-35, 2001.

13. GENOVESE WJ. Laser de baixa intensidade. 1. Ed. São Paulo: Lovise, 2000.

14. GENOVESE WJ. Laser de baixa intensidade: aplicações terapêuticas. 2. Ed. São Paulo: Santos, 2007.

15. GUZZARDELLA GA. et al. Assessment of low-power laser bioestimulation on chondral lesions: an "in vivo" experimental study. Artificial Cells, Blood Substitutes, and Biotechnology, Londres, v. 28, n. 5, p. 441-449, 2000.

16. HADDAD M F. et al. Conceitos básicos para a reabilitação oral por meio de implantes osseointegrados: parte I: influência do diâmetro e do comprimento. Revista Odontológica de Araçatuba (Online), São Paulo, v. 29, n. 1, p. 30$37,2008$.

17. JAKSE N. et al. Influence of low-level laser treatment on boné regeneration and osseointegration of dental implants following sinus augmentation: an experimental study on sheep. Clinical Oral Implants Research, Paris, v. 14, n. 4, p. 517-524, 2007.

18. JESUS LK. Avaliação dos efeitos do laser de baixa intensidade na osseointegração de implantes com diferentes superfícies: análise por frequência de ressonância e biomecânica. 2017. 43 f. Trabalho de Conclusão de Curso (Monografia). Faculdade de Odontologia de Araçatuba da Universidade Estadual Paulista "Júlio de Mesquita Filho". São Paulo, 2017.

19. LINS RDAU. et al. Aplicação do laser de baixa potência na cicatrização de feridas. Odontologia Clínico-Científica, Recife, v. 1, n. 1, p. 511-516, 2011.

20. LUÍS AA. Efeitos do laser de baixa potência no processo de cicatrização de feridas cutâneas: revisão de literatura. 2013. 35 f. Trabalho de conclusão de Curso (monografia). Centro Universitário de Formiga, Formiga, 2013.

21. MARIA MMA. E. Conhecimentos atuais sobre uso da terapia com laser em baixa intensidade para promoção do reparo ósseo. 2015. 32 f. Trabalho de Conclusão de Curso (Monografia). Universidade Estadual Paulista "Júlio de Mesquita Filho", Faculdade de Odontologia, Araçatuba, 2015.

22. MARTINS V. et al. Osseointegração: análise de fatores clínicos de sucesso e insucesso. Revista Odontológica de Araçatuba, v. 32, n. 1, p. 26-31, 2011.

23. MIRANDA TAC. et al. A influência do fumo na reabilitação com implantes osseointegrados: revisão de literatura. Revista de Odontologia da Universidade Cidade de São Paulo, São Paulo, v. 30, n. 2, p. 169-176, 2018.

24. PIRES-OLIVEIRA DDA. et al. Laser $904 \mathrm{~nm}$ action on bone repair in rats with osteoporosis. Osteoporosis International, Berlim, v. 21, n. 12, p. 2109-2114, 2010.

25. PROCKTA P, TAKAHASH A, PAGNONCELLI RM. Uso de terapia com laser de baixa intensidade na cirurgia bucomaxilofacial. Revista Portuguesa de Estomatologia, Medicina Dentária e Cirurgia Maxilofacial, Lisboa, v. 49, n. 4, p. 247-255, 2008.

26. SILVA FL et al. Tratamento de superfície em implantes dentários: uma revisão de literatura. Revista da Faculdade de Odontologia, Passo Fundo, v. 21, n. 1, p. 136-142, 2016.

27. TORRES MARO; ; TEIXEIRA ER. Influência do laser de baixa potência (GaAIAs - 830nm) na formação óssea em relação à estabilidade primária em osso tipo IV. Revista Odonto Ciência, Porto Alegre, v. 23, n. 2, p. 175-181, 2008.

28. XAVIER KCR.; SCHMIDT EMM.; SILVA G. Os efeitos do laser de baixa potência: um levantamento bibliográfico. In: Congresso de Engenharia Biomédica, n. 21, 2008, São José dos Campos. XXI Congresso de Engenharia Biomédica, São José dos Campos: Universidade do Vale do Paraíba.

29. YAMADA JÚNIOR A.; HAYEK R.; RIBEIRO M. O emprego da terapia fotodinâmica (PDT) na redução bacteriana em periodontia e implantodontia. Revista Gaúcha de Odontologia, Campinas, v. 52, n. 3, p. 207-210, 2004.

30. ZANCA MM. et al. Laserterapia de baixa intensidade: tratamento inovador na odontologia. Ação Odonto, Santa Catarina, v. 1, n. 1, p. 27, 2016. 